\title{
THE SECOND VARIATION OF A DEFINITE INTEGRAL.
}

\author{
BY DR. A. L. UNDERHILL.
}

(Read before the Chicago Section of the American Mathematical Society, January 1, 1908, and January 2, 1909.)

\section{Introduction.}

In the discussion of the minimizing of

$$
J=\int_{t_{0}}^{t_{1}} F\left(x, y, x^{\prime}, y^{\prime}\right) d t
$$

it may happen that the solution of the Jacobi equation presents considerable difficulty. In the following pages a method is given whereby without the solution of the Jacobi equation certain information may be obtained as to the extent of the interval in which a weak minimum exists.

It will be shown that by the evaluation of a quantity $\bar{K}_{0}$, which is computed along the extremal of the problem, one can draw the following conclusions :

1) If $\bar{K}_{0} \leqq 0$ along a given extremal arc, there exists no conjugate point on the arc, $i$. e., the extremal furnishes a weak minimum.

2) If $0<1 / \bar{K}_{0} \leqq a^{2}$ along an extremal, then the extremal cannot be a minimizing curve in any arc along which the value of the integral $J$ is greater than $\pi a$.

3 ) If $0<b^{2} \leqq 1 / \bar{K}_{0}$, then the extremal furnishes a weak minimum on any arc along which the value of $J$ is less than or equal to $\pi b$.

\section{§1. Proofs of the Preceding Theorems.}

During a study of invariants connected with the minimizing of (1), it was noted by the writer that the second variation of $J$ could be given the following invariantive normal form : *

$$
\delta^{2} J=\int_{\alpha_{0}}^{\alpha_{1}}\left[\left(\frac{d V}{d \alpha}\right)^{2}-\bar{K}_{0} V^{2}\right] d \alpha,
$$

\footnotetext{
* Transactions Amer. Math. Society, vol. 9 (1908), p. 336.
} 
in which

$$
\alpha=\int_{t_{0}}^{t} F\left(x, y, x^{\prime}, y^{\prime}\right) d t
$$

and $V$ and $\bar{K}_{0}$ are two absolute invariants under a point transformation

$$
x=X(u, v), \quad y=Y(u, v),
$$

as well as a parameter transformation $t=\chi(\tau)$; the defining equations for $V$ and $\overline{K_{0}}$ are

$$
\begin{gathered}
V=\omega F^{\frac{1}{2}}=u ; F^{\frac{1}{2}} F_{1}^{\frac{1}{2}}=\left(y^{\prime} \delta x+x^{\prime} \delta y\right) F^{\frac{1}{2}} F_{1}^{\frac{1}{2}} * \\
\bar{K}_{0}=\frac{1}{F^{2}}\left[\frac{1}{4} \frac{F_{1}^{\prime 2}}{F_{1}^{2}}-\frac{1}{2} \frac{F_{1}^{\prime \prime}}{F_{1}^{\prime}}-\frac{F_{2}}{F_{1}^{\prime}}-\frac{1}{2} \frac{F^{\prime \prime}}{F}+\frac{3}{4} \frac{F^{\prime 2}}{F^{2}}\right]{ }^{\dagger}
\end{gathered}
$$

From the above form (2) of the second variation follows at once :

Theorem $A$ : In case $\bar{K}_{0} \leqq 0$ along an extremal are, it follows thal $\delta^{2} J>0$, and the extremal are furnishes a weak minimum. $\$$

In order to obtain the other results, we start from (2) and form its Jacobi equation $\S$

$$
\psi(V)=-\bar{K}_{0} V-\frac{d^{2} V}{d \alpha^{2}}=0
$$

and denote by $V=\Phi(\alpha)$ the solution of the equation which vanishes for $\alpha=\alpha_{0}$. The next zero of $\Phi(\alpha)$ may often be approximately located by comparing it with the solution of the equation

$$
\frac{d^{2} V}{d \alpha^{2}}+\frac{1}{a^{2}} V=0
$$

which vanishes at $\alpha=\alpha_{0}$,

$$
V=c \sin \left(\frac{\alpha-\alpha_{0}}{a}\right) \text {. }
$$

The next zero of this solution is at $\alpha=\alpha_{0}+\pi \alpha$.

Use may now be made of the following theorems of Sturm, $\|$

* Transactions, loc. cit., p. 336, p. 329, p. 327.

† Ibid., p. 334.

$\ddagger$ Ibid., p. 336.

\$ Cf. Bolza, Lectures on the calculus of variations, p. 133.

|| Darboux, Théorie des surfaces, \$629. 
regarding the solutions of the two differential equations

$$
\frac{d^{2} V}{d x^{2}}=H V, \quad \frac{d^{2} V}{d x^{2}}=H^{\prime} V .
$$

1) If $V=\phi(x)$ is a solution of the first equation having consecutive zeros at $x_{0}$ and $x_{1}$, and if $H^{\prime} \geqq H$ for all values of $x$ in the interval $\left(x_{0}, x_{1}\right)$, then the solution of the second which vanishes at $x_{0}$ does not vanish again within or at the end point $x_{1}$ of the interval $\left(x_{0}, x_{1}\right)$.

2) If $H^{\prime} \leqq H$ for all values of $x$ in $\left(x_{0}, x_{1}\right)$, then the solution of the second which vanishes for $x=x_{0}$ has at least one zero in the interval $\left(x_{0}, x_{1}\right)$.

Using (5) and (4) as the two equations of (6), we can formulate the following:

THEOREM $B:$ If $1 / \bar{K}_{0}$ is positive and greater than $b^{2}$ along an extremal, then any arc of the extremal along which the value of $J$ $i s \leqq \pi b$ furnishes a weak minimum to $J$.

Theorem $C:$ If $1 / \overline{K_{0}}$ is positive and less than $a^{2}$ along the extremal, then no arc of the extremal along which the value of $J$ is greater than $\pi$ a can be a minimizing curve.

Comparing these theorems, we notice that $A$ is applicable in case $\bar{K}_{0}$ is $\leqq 0$, while $B$ and $C$ apply when $\bar{K}_{0}$ is $\geqq 0$, the former when $1 / \bar{K}_{0}$ is greater, the latter when $1 / \bar{K}_{0}$ is smaller, than some definite constant.

\section{§ 2. Applications.}

Since $\bar{K}_{0}$ is an invariant under both point and parameter transformation the computational work is simplified by properly choosing the coordinate system as well as the parameter of the extremal.

1) The geodesic problem.*

We choose as the $u, v$ coordinates of the surface the geodesic parallel coordinates and for parameter the arc of the curve on the surface. $\bar{K}_{0}$ turns out to be the gaussian curvature, and we have, when use is made of theorem $A$, the well known Jacobi-Bonnet theorem :

On a surface of negative curvature, a given point has no conjugate point on the geodesics which pass through it.

* Result already given in Transactions, loc. cit., p. 337 . 
2) The problem of the shortest distance between two points.

By choosing the arc as the parameter, the extremal may be written in the form

$$
x=\alpha+\beta s, \quad y=\gamma+\delta s,
$$

$\alpha, \beta, \gamma$, and $\delta$ being constants.

The computation of the several quantities which appear in $\bar{K}_{0}$, each one being taken along the extremal, leads to the following :

$$
\begin{aligned}
& F=1, \quad F^{\prime}=0, \quad F^{\prime \prime}=0, \\
& F_{1}=1, \quad F_{1}^{\prime}=0, \quad F_{1}^{\prime \prime}=0, \\
& F_{2}=x^{\prime} x^{\prime \prime \prime}+y^{\prime} y^{\prime \prime \prime}=0 .
\end{aligned}
$$

As a result, $\bar{K}_{0}=0$ and $1 / \bar{K}_{0}=\infty>b^{2}$ for any tinite $b$, and by means of theorem $B$ we have :

For the problem of finding the shortest distance between two points, the extremal

$$
x=\alpha+\beta s, \quad y=\gamma+\delta s,
$$

furnishes a weak minimum in any interval.

3) The problem of the minimum surface of revolution.

The extremal is in this case a catenary and we choose the coordinate system so that the curve is symmetrical with respect to the $y$-axis, with its lowest point at $(0, a)$. If the arc $s$ is selected as the parameter, then the equation of the extremal is

$$
x=a \operatorname{arcsinh}\left(\frac{s}{a}\right), \quad y=\frac{a}{2}\left[e^{\operatorname{arcsinh}\left(\frac{s}{a}\right)}+e^{-\operatorname{arcsinh}\left(\frac{s}{a}\right)}\right],
$$

since $s=a \sinh (s / a)$.

The computation leads to the following:

$$
\begin{aligned}
& F=y, \quad F^{\prime}=y^{\prime}, \quad F^{\prime \prime}=y^{\prime \prime}, \\
& F_{1}=y, \quad F_{1}^{\prime \prime}=y^{\prime}, \quad F_{1}^{\prime \prime}=y^{\prime \prime}, \\
& F_{2}^{\prime}=-y^{\prime \prime}-y\left(x^{\prime} y^{\prime \prime}-x^{\prime \prime} y^{\prime}\right)^{2}=-y^{\prime \prime}-\frac{y}{\rho^{2}},
\end{aligned}
$$

where $\rho$ is the radius of curvature for the catenary. Since $1 / \rho=\alpha / y^{2}$, one obtains upon substitution of these values in $\bar{K}_{0}$ 
since $y \geqq a$.

$$
\bar{K}_{0} \doteq \frac{1}{y^{4}} \leqq \frac{1}{a^{4}}, \quad \frac{1}{\bar{K}_{0}} \geqq a^{4},
$$

The use of theorem $B$ leads to the following statement:

For the problem of the minimum surface of revolution, an arc of the catenary along which the value of $J$ is at most equal to $\pi a^{2}$ furnishes a weak minimum.

In case $\left(x_{0}, y_{0}\right)$ is the first point of the arc of the extremal, the abscissa $\bar{x}$ of the end point of the arc which will satisfy the above condition is given by the equation

$$
\sinh \left(\frac{2 x}{a}\right)+2 \bar{x}-\left[\sinh \left(\frac{2 x_{0}}{a}\right)+2 x_{0}\right]=4 \pi a .
$$

4) The brachistochrone problem.

The extremal is a cycloid and the origin is chosen at a cusp, the $y$ axis is directed downward, and the arc $s$ is selected as the parameter.

From the usual equation of the cycloid by means of the relation

$$
s=4 a\left(1-\cos \frac{1}{2} \theta\right)
$$

we obtain the equation of the extremal under the present choice of axis and parameter as

$$
\begin{gathered}
x=a\left[\sin ^{-1}\left(2 \sqrt{\frac{s}{2 a}-\frac{s^{2}}{16 a}} \cdot \frac{4 a-s}{4 a}\right)-2\left(2 \sqrt{\frac{s}{2 a}-\frac{s^{2}}{16 a}} \cdot \frac{4 a-s}{4 a}\right)\right], \\
y=s-\frac{s^{2}}{8 a} .
\end{gathered}
$$

The computation gives the following values:

$$
\begin{aligned}
& F^{\prime}=\frac{1}{\sqrt{y}}, \quad F^{\prime}=-\frac{1}{2} \frac{y^{\prime}}{\sqrt{y^{3}}}, \quad F^{\prime \prime}=\frac{3}{4} \frac{y^{\prime 2}}{\sqrt{y^{5}}}-\frac{1}{2} \frac{y^{\prime \prime}}{\sqrt{y^{3}}}, \\
& F_{1}=\frac{1}{\sqrt{y}}, \quad F_{1}^{\prime}=-\frac{1}{2} \frac{y^{\prime}}{\sqrt{y^{3}}}, \quad F_{1}^{\prime \prime}=\frac{3}{4} \frac{y^{\prime 2}}{\sqrt{y^{5}}}-\frac{1}{2} \frac{y^{\prime \prime}}{\sqrt{y^{3}}}, \\
& F_{2}=\frac{3}{4} \frac{1}{\sqrt{y^{5}}}+\frac{1}{2} \frac{y^{\prime \prime}}{\sqrt{y^{3}}}-\frac{3}{4} \frac{y^{\prime 2}}{\sqrt{y^{5}}}-\frac{1}{\rho^{2} \sqrt{y}} .
\end{aligned}
$$


For the cycloid

$$
\frac{1}{\rho^{2}}=\frac{1}{16 a^{2} \sin ^{2} \frac{1}{2} \bar{\theta}}=\frac{1}{8 a\left(s-\frac{s^{2}}{8 a}\right)}=\frac{1}{8 a y},
$$

and after substitution of these values in $\bar{K}_{0}$ we find

$$
\bar{K}_{0}=\frac{1}{8 a y}\left\{2 a\left(y^{\prime 2}-3\right)+y\right\}
$$

or making use of the extremal equation,

$$
\bar{K}_{0}=-\frac{1}{2} y, \text { i. e., } \quad \bar{K}_{0}<0 .
$$

By means of theorem $A$, we have the result:

For the brachistochrone problem there is no conjugate point to any point $P$ lying on the same cycloid arch with $P$.

The University of Wisconsin.

\section{A SIMPLER PROOF OF LIE'S THEOREM FOR ORDINARY DIFFERENTIAL EQUATIONS.}

BY PROFEssor L. D. AMES.

(Read before the Chicago Section of the American Mathematical Society, April 9, 1909.)

The following theorem is essentially equivalent to Lie's principal theorem concerning the integration of the differential equation $\Omega\left(x, y, y^{\prime}\right)=0$ when it is invariant under a known group. As stated here, this theorem makes no use of the idea of a group.

Theorem. Given any differential equation of the form

$$
\Omega\left(x, y, y^{\prime}\right)=0
$$

which can be solved in the form

$$
X(x, y) y^{\prime}-Y(x, y)=0 ;
$$

if $\xi(x, y)$ and $\eta(x, y)$ are such functions that

$$
X \eta-Y \xi \neq 0,
$$

